\title{
Microwave-Assisted One-Pot Synthesis of 1-Indanones from Arenes and $\alpha, \beta$-Unsaturated Acyl Chlorides
}

\author{
Wei Yin ${ }^{a}$, Yuan $\mathrm{Ma}^{a}{ }^{*}{ }^{*}$ Jiaxi Xu ${ }^{b}$, and Yufen Zhao ${ }^{a}$
}

\begin{abstract}
${ }^{a}$ Key Laboratory of Bioorganic Phosphorus Chemistry and Chemical Biology, Ministry of
Education, Department of Chemistry, Tsinghua University, Beijing 100084, People's

Republic of China. ${ }^{b}$ Key Laboratory of Bioorganic Chemistry and Molecular Engineering of Ministry of Education, College of Chemistry and Molecular Engineering, Peking University, Beijing 100871, People's Republic of China

mayuan@mail.tsinghua.edu.cn
\end{abstract}

\section{Supplemental Materials}

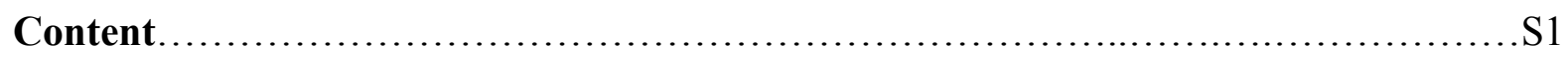

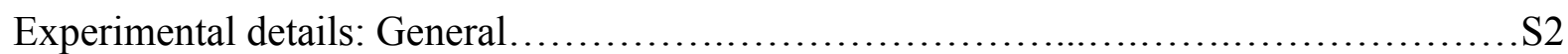

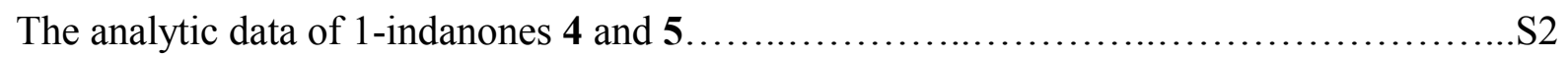

Copies of ${ }^{1} \mathrm{H}$ NMR and ${ }^{13} \mathrm{C}$ NMR spectra of the unknown compounds 4 and $\mathbf{5} \ldots \ldots \ldots \ldots$........ 8

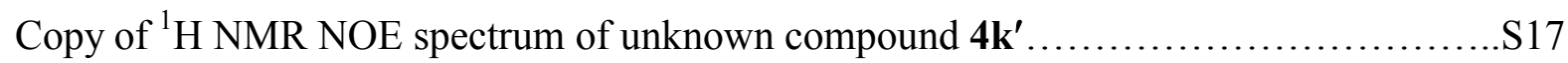




\section{Experimental Details}

General. $o$-Dichlorobenzene was dried over anhydrous sodium sulfate and distilled prior to use. All reactions were performed in a commercial microwave reactor (XH-100A, $100-1000$ W, Beijing Xianghu Science and Technology Development Co. Ltd, Beijing, P. R. China). The temperature of the reaction mixture was measured by an immersed platinum resistance thermometer. Melting points were measured on a melting point apparatus and are uncorrected. ${ }^{1} \mathrm{H}$ NMR (200 or $300 \mathrm{MHz}$ ) and ${ }^{13} \mathrm{C}$ NMR (50 or $75 \mathrm{MHz}$ ) spectra were recorded in $\mathrm{CDCl}_{3}$ with TMS as an internal standard. Mass spectra and HRMS data were obtained on an EI mass spectrometer. IR spectra were recorded on a FT-IR spectrometer with an OMNI sampler. TLC separations were performed on silica gel $\mathrm{GF}_{254}$ plates, and the plates were visualized with UV light.

\section{3-Methyl-1-indanone (4a)}<smiles>CC1CC(=O)c2ccccc21</smiles>

Colorless oil, ${ }^{1} \mathrm{H}$ NMR (300 MHz, $\left.\mathrm{CDCl}_{3}\right) \delta 7.73-7.34$ (m, 4H), 3.43 (ddq, $\left.J=7.5,3.4,6.9 \mathrm{~Hz}, 1 \mathrm{H}\right), 2.93$ $(\mathrm{dd}, J=19.2,7.5 \mathrm{~Hz}, 1 \mathrm{H}), 2.27(\mathrm{dd}, J=19.2,3.4 \mathrm{~Hz}, 1 \mathrm{H}), 1.40(\mathrm{~d}, J=6.9 \mathrm{~Hz}, 3 \mathrm{H}) ;{ }^{13} \mathrm{C}$ NMR $(75.5 \mathrm{MHz}$, $\left.\mathrm{CDCl}_{3}\right) \delta 21.2,32.6,45.2,123.3,125.2,127.3,134.6,136.3,159.8,206.3$. Known compound. ${ }^{1}$

\section{3,5-Dimethyl-1-indanone (4b)}<smiles>Cc1ccc2c(c1)C(C)CC2=O</smiles>

Colorless oil, ${ }^{1} \mathrm{H}$ NMR $\left(200 \mathrm{MHz}, \mathrm{CDCl}_{3}\right) \delta 7.61(\mathrm{~d}, J=8.0 \mathrm{~Hz}, 1 \mathrm{H}), 7.29(\mathrm{~s}, 1 \mathrm{H}), 7.17(\mathrm{~d}, J=8.0 \mathrm{~Hz}, 1 \mathrm{H})$, 3.38 (ddq, $J=7.6,3.5,7.4 \mathrm{~Hz}, 1 \mathrm{H}), 2.91$ (dd, $J=19.0,7.6 \mathrm{~Hz}, 1 \mathrm{H}), 2.45$ (s, 3H), 2.24 (dd, $J=19.0,3.5 \mathrm{~Hz}$, $1 \mathrm{H}), 1.38(\mathrm{~d}, J=7.4 \mathrm{~Hz}, 3 \mathrm{H}) ;{ }^{13} \mathrm{C}$ NMR $\left(50.3 \mathrm{MHz}, \mathrm{CDCl}_{3}\right) \delta 21.2,22.0,32.5,45.4,123.2,125.5,128.6$, 134.1, 145.8, 160.4, 205.6. Known compound. ${ }^{2}$

\section{5-Fluoro-3-methyl-1-indanone (4c)}<smiles>CC1CC(=O)c2ccc(F)cc21</smiles>

Colorless oil, ${ }^{1} \mathrm{H}$ NMR $\left(200 \mathrm{MHz}, \mathrm{CDCl}_{3}\right) \delta$ 7.76-7.69 (m, 1H), 7.18-7.02 (m, 2H), 3.43 (ddq, $J=7.4,3.4$, $7.0, \mathrm{~Hz}, 1 \mathrm{H}), 2.96(\mathrm{dd}, J=18.9,7.4 \mathrm{~Hz}, 1 \mathrm{H}), 2.30(\mathrm{dd}, J=18.9,3.4 \mathrm{~Hz}, 1 \mathrm{H}), 1.41(\mathrm{~d}, J=7.0 \mathrm{~Hz}, 3 \mathrm{H}) ;{ }^{13} \mathrm{C}$ NMR $\left(50.3 \mathrm{MHz}, \mathrm{CDCl}_{3}\right) \delta 21.1,32.6,45.4,124.9,125.6,127.4,132.8,155.0,162.9,204.3$. Known compound. $^{4}$

\section{5-Chloro-3-methyl-1-indanone (4d)}


<smiles>CC1CC(=O)c2ccc(Cl)cc21</smiles>

Colorless oil, ${ }^{1} \mathrm{H}$ NMR $\left(200 \mathrm{MHz}, \mathrm{CDCl}_{3}\right) \delta 7.63(\mathrm{~d}, J=8.4 \mathrm{~Hz}, 1 \mathrm{H}), 7.49(\mathrm{~s}, 1 \mathrm{H}), 7.34(\mathrm{~d}, J=8.4 \mathrm{~Hz}, 1 \mathrm{H})$, $3.42(\mathrm{ddq}, J=7.6,3.6,7.0 \mathrm{~Hz}, 1 \mathrm{H}), 2.95(\mathrm{dd}, J=19.2,7.6 \mathrm{~Hz}, 1 \mathrm{H}), 2.28(\mathrm{dd}, J=19.2,3.6 \mathrm{~Hz}, 1 \mathrm{H}), 1.40$ (d, $J=7.0 \mathrm{~Hz}, 3 \mathrm{H}) ;{ }^{13} \mathrm{C}$ NMR $\left(50.3 \mathrm{MHz}, \mathrm{CDCl}_{3}\right) \delta 21.0,32.6,45.2,124.6,125.5,127.4,128.1,141.2,161.3$, 204.8. Known compound. ${ }^{5}$

\section{5-Bromo-3-methyl-1-indanone (4e)}<smiles>CC1CC(=O)c2ccc(Br)cc21</smiles>

Colorless oil, ${ }^{1} \mathrm{H}$ NMR $\left(300 \mathrm{MHz}, \mathrm{CDCl}_{3}\right) \delta 7.64(\mathrm{~d}, J=8.2 \mathrm{~Hz}, 1 \mathrm{H}), 7.56(\mathrm{~s}, 1 \mathrm{H}), 7.51(\mathrm{~d}, J=8.2 \mathrm{~Hz}, 1 \mathrm{H})$, $3.43(\mathrm{ddq}, J=7.7,3.6,7.2 \mathrm{~Hz}, 1 \mathrm{H}), 2.93(\mathrm{dd}, J=19.2,7.7 \mathrm{~Hz}, 1 \mathrm{H}), 2.27$ (dd, $J=19.2,3.6 \mathrm{~Hz}, 1 \mathrm{H}), 1.40$ (d, $J=7.2 \mathrm{~Hz}, 3 \mathrm{H}) ;{ }^{13} \mathrm{C} \mathrm{NMR}\left(75.5 \mathrm{MHz}, \mathrm{CDCl}_{3}\right) \delta 21.0,32.5,45.1,124.5,128.6,129.9,130.9,135.1,161.3$, 204.7. Known compound. ${ }^{6}$

\section{3-Methyl-5-phenyl-1-indanone (4f)}<smiles>CC1CC(=O)c2ccc(-c3ccccc3)cc21</smiles>

Colorless crystals, m.p. $79.5-80.5{ }^{\circ} \mathrm{C}$; $\mathrm{Lit}^{7}$ m.p. $74-75.5{ }^{\circ} \mathrm{C} ;{ }^{1} \mathrm{H}$ NMR $\left(300 \mathrm{MHz}, \mathrm{CDCl}_{3}\right) \delta$ 7.82-7.42 (m, $8 \mathrm{H}), 3.50$ (ddq, $J=7.6,3.4,7.2 \mathrm{~Hz}, 1 \mathrm{H}), 2.99(\mathrm{dd}, J=19.0,7.6 \mathrm{~Hz}, 1 \mathrm{H}), 2.34(\mathrm{dd}, J=19.0,3.4 \mathrm{~Hz}, 1 \mathrm{H})$, $1.46(\mathrm{~d}, J=7.2 \mathrm{~Hz}, 3 \mathrm{H}) ;{ }^{13} \mathrm{C} \mathrm{NMR}\left(75.5 \mathrm{MHz}, \mathrm{CDCl}_{3}\right) \delta 21.3,32.8,45.6,123.77,123.80,126.9,127.5$, $128.3,128.9,135.2,140.3,147.9,160.6,206.1$.

\section{5-Isopropyl-3-methyl-1-indanone (4g)}<smiles>CC(C)c1ccc2c(c1)C(C)CC2=O</smiles>

Colorless oil, $\mathrm{R}_{f}=0.38$ [ethyl acetate: petroleum ether $\left(60-90^{\circ} \mathrm{C}\right) 1: 15$, v/v, silica gel plate]; IR $(\mathrm{KBr}) v$ $\left(\mathrm{cm}^{-1}\right): 1711.8(\mathrm{C}=\mathrm{O}) ;{ }^{1} \mathrm{H}$ NMR $\left(200 \mathrm{MHz}, \mathrm{CDCl}_{3}\right) \delta 7.65(\mathrm{~d}, J=8.0 \mathrm{~Hz}, 1 \mathrm{H}), 7.34(\mathrm{~s}, 1 \mathrm{H}), 7.25(\mathrm{~d}, J=8.0$ $\mathrm{Hz}, 1 \mathrm{H}), 3.40$ (ddq, $J=7.4,3.4,7.0 \mathrm{~Hz}, 1 \mathrm{H}), 2.98(\mathrm{~m}, J=7.0 \mathrm{~Hz}, 1 \mathrm{H}), 2.92(\mathrm{dd}, J=19.0,7.4 \mathrm{~Hz}, 1 \mathrm{H}), 2.26$ $(\mathrm{dd}, J=19.0,3.4 \mathrm{~Hz}, 1 \mathrm{H}), 1.40(\mathrm{~d}, J=7.0 \mathrm{~Hz}, 3 \mathrm{H}), 1.29(\mathrm{~d}, J=7.0 \mathrm{~Hz}, 6 \mathrm{H}) ;{ }^{13} \mathrm{C} \mathrm{NMR}\left(50.3 \mathrm{MHz}, \mathrm{CDCl}_{3}\right)$ $\delta 21.2,23.6,32.6,34.6,45.5,122.8,123.3,126.1,134.4,156.7,160.5,205.9$. MS (EI) $\mathrm{m} / z$ (rel. intensity, \%): $188\left(\mathrm{M}^{+}, 44\right), 173\left(\mathrm{M}^{+}-\mathrm{CH}_{3}, 100\right), 145\left(\mathrm{M}^{+}-\mathrm{Pr}, 29\right), 131$ (22), 115 (21), 77 (11); HRMS Cacld. for $\mathrm{C}_{13} \mathrm{H}_{16} \mathrm{O}\left(\mathrm{M}^{+}\right)$188.1201; Found 188.1201. 
<smiles>Cc1cc(C)c2c(c1)C(C)CC2=O</smiles>

Colorless oil, ${ }^{1} \mathrm{H}$ NMR (300 MHz, $\left.\mathrm{CDCl}_{3}\right) \delta 7.11(\mathrm{~s}, 1 \mathrm{H}), 6.92(\mathrm{~s}, 1 \mathrm{H}), 3.31$ (ddq, $J=7.8,3.6,7.2 \mathrm{~Hz}, 1 \mathrm{H}$ ), $2.88(\mathrm{dd}, J=18.9,7.8 \mathrm{~Hz}, 1 \mathrm{H}), 2.59(\mathrm{~s}, 3 \mathrm{H}), 2.39(\mathrm{~s}, 3 \mathrm{H}), 2.23(\mathrm{dd}, J=18.9,3.6 \mathrm{~Hz}, 1 \mathrm{H}), 1.35(\mathrm{~d}, J=7.2$ $\mathrm{Hz}, 3 \mathrm{H}) ;{ }^{13} \mathrm{C} \mathrm{NMR}\left(75.5 \mathrm{MHz}, \mathrm{CDCl}_{3}\right) \delta 18.2,21.4,21.8,31.9,46.0,123.0,130.3,131.6,138.2,145.0$, 161.3, 206.7. Known compound. ${ }^{2}$

\section{3,4,7-Trimethyl-1-indanone $(4 \mathbf{j})$}<smiles>Cc1ccc(C)c2c1C(=O)CC2C</smiles>

Colorless oil, ${ }^{1} \mathrm{H}$ NMR $\left(300 \mathrm{MHz}, \mathrm{CDCl}_{3}\right) \delta 7.23(\mathrm{~d}, J=7.5 \mathrm{~Hz}, 1 \mathrm{H}), 7.01(\mathrm{~d}, J=7.5 \mathrm{~Hz}, 1 \mathrm{H}), 3.43$ (ddq, $J$ $=7.8,1.6,6.9 \mathrm{~Hz}, 1 \mathrm{H}), 2.88(\mathrm{dd}, J=18.7,7.8 \mathrm{~Hz}, 1 \mathrm{H}), 2.59(\mathrm{~s}, 3 \mathrm{H}), 2.36(\mathrm{~s}, 3 \mathrm{H}), 2.29(\mathrm{dd}, J=18.7,1.6 \mathrm{~Hz}$, $1 \mathrm{H}), 1.29(\mathrm{~d}, J=6.9 \mathrm{~Hz}, 3 \mathrm{H}) ;{ }^{13} \mathrm{C} \mathrm{NMR}\left(75.5 \mathrm{MHz}, \mathrm{CDCl}_{3}\right) \delta 17.6,18.0,21.3,31.4,46.3,129.4,132.3$, 133.2, 135.3, 135.8, 158.8, 207.8. Known compound. ${ }^{8}$

\section{5,6-Dichloro-3-methyl-1-indanone (4k)}<smiles>CC1CC(=O)c2cc(Cl)c(Cl)cc21</smiles>

Yellowish solid, m.p. $67.5-68.5{ }^{\circ} \mathrm{C} ; \mathrm{R}_{f}=0.26$ [ethyl acetate: petroleum ether $\left(60-90{ }^{\circ} \mathrm{C}\right) 1: 15$, v/v, silica gel plate]; IR $(\mathrm{KBr}) v\left(\mathrm{~cm}^{-1}\right): 1720.2(\mathrm{C}=\mathrm{O}) ;{ }^{1} \mathrm{H}$ NMR $\left(200 \mathrm{MHz}, \mathrm{CDCl}_{3}\right) \delta 7.79(\mathrm{~s}, 1 \mathrm{H}), 7.62(\mathrm{~s}, 1 \mathrm{H}), 3.42$ (ddq, $J=7.6,3.2,7.0 \mathrm{~Hz}, 1 \mathrm{H}), 2.98$ (dd, $J=19.2,7.6 \mathrm{~Hz}, 1 \mathrm{H}), 2.32$ (dd, $J=19.2,3.2 \mathrm{~Hz}, 1 \mathrm{H}), 1.41$ (d, $J=$ $7.0 \mathrm{~Hz}, 3 \mathrm{H}) ;{ }^{13} \mathrm{C} \mathrm{NMR}\left(50.3 \mathrm{MHz}, \mathrm{CDCl}_{3}\right) \delta 21.1,32.4,45.3,116.0,124.9,127.4,136.0,139.2,158.6$, 203.7. MS (EI) $m / z$ (rel. intensity, \%): $214\left(\mathrm{M}^{+}, 56\right), 199\left(\mathrm{M}^{+}-\mathrm{CH}_{3}, 100\right), 179\left(\mathrm{M}^{+}-\mathrm{Cl}, 15\right), 136(20), 115$ (30), 83 (16); HRMS Cacld. for $\mathrm{C}_{10} \mathrm{H}_{8} \mathrm{Cl}_{2} \mathrm{O}\left(\mathrm{M}^{+}\right)$213.9952; Found 213.9952.

\section{4,5-Dichloro-3-methyl-1-indanone (4k')}<smiles>CC1CC(=O)c2c1ccc(Cl)c2Cl</smiles>

Yellowish oil, $\mathrm{R}_{f}=0.34$ [ethyl acetate: petroleum ether $\left(60-90{ }^{\circ} \mathrm{C}\right) 1: 15$, v/v, silica gel plate]; IR $(\mathrm{KBr}) v$ $\left(\mathrm{cm}^{-1}\right): 1721.0(\mathrm{C}=\mathrm{O}) ;{ }^{1} \mathrm{H}$ NMR $\left(200 \mathrm{MHz}, \mathrm{CDCl}_{3}\right) \delta 7.56(\mathrm{~d}, J=8.1 \mathrm{~Hz}, 1 \mathrm{H}), 7.48(\mathrm{~d}, J=8.1 \mathrm{~Hz}, 1 \mathrm{H})$, 3.60 (ddq, $J=7.6,1.2,6.8 \mathrm{~Hz}, 1 \mathrm{H}$ ), 2.99 (dd, $J=19.2,7.6 \mathrm{~Hz}, 1 \mathrm{H}$ ), 2.40 (dd, $J=19.2,1.2 \mathrm{~Hz}, 1 \mathrm{H}$ ), 1.44 (d, $J=6.8 \mathrm{~Hz}, 3 \mathrm{H}) ;{ }^{13} \mathrm{C} \mathrm{NMR}\left(50.3 \mathrm{MHz}, \mathrm{CDCl}_{3}\right) \delta 20.2,33.0,45.6,122.2,130.1,130.7,136.2,139.5,158.1$, 204.1. MS (EI) $m / z$ (rel. intensity, \%): $214\left(\mathrm{M}^{+}, 46\right), 199\left(\mathrm{M}^{+}-\mathrm{CH}_{3}, 100\right), 179\left(\mathrm{M}^{+}-\mathrm{Cl}, 9\right), 136$ (14), 115 (22), 83 (19); HRMS Cacld. for $\mathrm{C}_{10} \mathrm{H}_{8} \mathrm{Cl}_{2} \mathrm{O}\left(\mathrm{M}^{+}\right)$214.9952; Found 214.9949. 


\section{3-Phenyl-1-indanone (4l)}<smiles>O=C1CC(c2ccccc2)c2ccccc21</smiles>

Colorless crystals, m.p. $77-78.5{ }^{\circ} \mathrm{C}$; $\mathrm{Lit}^{1}$ m.p. $78{ }^{\circ} \mathrm{C} ;{ }^{1} \mathrm{H}$ NMR $\left(200 \mathrm{MHz}, \mathrm{CDCl}_{3}\right) \delta 7.62-7.11(\mathrm{~m}, 9 \mathrm{H}), 4.58$ (dd, $J=8.1,3.8 \mathrm{~Hz}, 1 \mathrm{H}), 3.24(\mathrm{dd}, J=19.2,8.1 \mathrm{~Hz}, 1 \mathrm{H}) 2.69(\mathrm{dd}, J=19.2,3.8 \mathrm{~Hz}, 1 \mathrm{H}) ;{ }^{13} \mathrm{C}$ NMR $(50.3$ $\left.\mathrm{MHz}, \mathrm{CDCl}_{3}\right) \delta 44.4,46.8,123.4,126.9,127.0,127.7,127.9,128.9,135.1,136.8,143.7,158.0,206.2$.

\section{5-Fluoro-3-phenyl-1-indanone (4m)}<smiles>O=C1CC(c2ccccc2)c2cc(F)ccc21</smiles>

Colorless crystals, m.p. $107.5-108.5{ }^{\circ} \mathrm{C} ; \mathrm{R}_{f}=0.30$ [ethyl acetate: petroleum ether $\left(60-90{ }^{\circ} \mathrm{C}\right) 1: 15, \mathrm{v} / \mathrm{v}$, silica gel plate]; IR $(\mathrm{KBr}) v\left(\mathrm{~cm}^{-1}\right): 1713.1(\mathrm{C}=\mathrm{O}) ;{ }^{1} \mathrm{H}$ NMR $\left(300 \mathrm{MHz}, \mathrm{CDCl}_{3}\right): \delta 7.83-6.95(\mathrm{~m}, 8 \mathrm{H}), 4.57$ (dd, $J=8.1,3.8 \mathrm{~Hz}, 1 \mathrm{H}), 3.23(\mathrm{dd}, J=19.3,8.1 \mathrm{~Hz}, 1 \mathrm{H}), 2.63(\mathrm{dd}, J=19.3,3.8 \mathrm{~Hz}, 1 \mathrm{H}) ;{ }^{13} \mathrm{C}$ NMR $(50$ $\mathrm{MHz}, \mathrm{CDCl}_{3}$ ): $\delta 43.7,46.9,115.5,115.9,123.5,126.7,128.0,129.0,129.2,135.1,157.6,164.3,205.4$. MS (EI) $m / z$ (rel. intensity, \%): $226\left(\mathrm{M}^{+}, 100\right), 208\left(\mathrm{M}^{+}-\mathrm{PhH}, 31\right), 207\left(\mathrm{M}^{+}-\mathrm{F}, 11\right), 197$ (39), 183 (31), 77 (24); HRMS Cacld. for $\mathrm{C}_{15} \mathrm{H}_{11} \mathrm{FO}\left(\mathrm{M}^{+}\right)$226.0794; Found 226.0788.

\section{5-Chloro-3-phenyl-1-indanone (4n)}<smiles>O=C1CC(c2ccccc2)c2cc(Cl)ccc21</smiles>

Colorless oil; ${ }^{1} \mathrm{H}$ NMR $\left(200 \mathrm{MHz}, \mathrm{CDCl}_{3}\right) \delta 7.03-7.83(\mathrm{~m}, 8 \mathrm{H}), 4.56(\mathrm{dd}, J=8.0,4.0 \mathrm{~Hz}, 1 \mathrm{H}), 3.22(\mathrm{dd}, J$ $=19.2,8.0 \mathrm{~Hz}, 1 \mathrm{H}) 2.62(\mathrm{dd}, J=19.2,4.0 \mathrm{~Hz}, 1 \mathrm{H}) ;{ }^{13} \mathrm{C} \mathrm{NMR}\left(50.3 \mathrm{MHz}, \mathrm{CDCl}_{3}\right) \delta 43.8,46.7,123.5$, $126.7,128.1,128.9,129.0,130.9,132.8,135.1,142.2,155.0,205.2$. Known compound. ${ }^{3,9}$

\section{3,7-Dimethyl-1-indanone (5b)}<smiles>Cc1cccc2c1C(=O)CC2C</smiles>

Colorless oil, ${ }^{1} \mathrm{H}$ NMR $\left(200 \mathrm{MHz}, \mathrm{CDCl}_{3}\right) \delta 7.44(\mathrm{t}, J=7.8,7.4 \mathrm{~Hz}, 1 \mathrm{H}), 7.25(\mathrm{~d}, J=7.8 \mathrm{~Hz}, 1 \mathrm{H}), 7.09$ (d, $J=7.4 \mathrm{~Hz}, 1 \mathrm{H}), 3.36$ (ddq, $J=7.7,3.6,7.0 \mathrm{~Hz}, 1 \mathrm{H}), 2.89$ (dd, $J=18.8,7.7 \mathrm{~Hz}, 1 \mathrm{H}), 2.63(\mathrm{~s}, 3 \mathrm{H}), 2.24$ (dd, $J=18.8,3.6 \mathrm{~Hz}, 1 \mathrm{H}), 1.37(\mathrm{~d}, J=7.0 \mathrm{~Hz}, 3 \mathrm{H}) ;{ }^{13} \mathrm{C} \mathrm{NMR}\left(50.3 \mathrm{MHz}, \mathrm{CDCl}_{3}\right) \delta 18.2,21.4,32.1,45.8$, $122.5,128.3,129.1,133.9,138.5,160.7,205.6$. Known compound. ${ }^{2,3}$ 


\section{7-Fluoro-3-methyl-1-indanone (5c)}<smiles>CC1CC(=O)c2c(F)cccc21</smiles>

Colorless oil, $\mathrm{R}_{f}=0.45$ [ethyl acetate: petroleum ether $\left(60-90{ }^{\circ} \mathrm{C}\right) 1: 15, \mathrm{v} / \mathrm{v}$, silica gel plate]; $\mathrm{IR}(\mathrm{KBr}) v$ $\left(\mathrm{cm}^{-1}\right): 1719.1(\mathrm{C}=\mathrm{O}) ;{ }^{1} \mathrm{H}$ NMR $\left(200 \mathrm{MHz}, \mathrm{CDCl}_{3}\right) \delta 7.59-7.09(\mathrm{~m}, 3 \mathrm{H}), 3.60(\mathrm{ddq}, J=7.8,1.6,7.0 \mathrm{~Hz}$, $1 \mathrm{H}), 2.99(\mathrm{dd}, J=19.2,7.8 \mathrm{~Hz}, 1 \mathrm{H}), 2.40(\mathrm{dd}, J=19.2,1.6 \mathrm{~Hz}, 1 \mathrm{H}), 1.44(\mathrm{~d}, J=7.0 \mathrm{~Hz}, 3 \mathrm{H}) ;{ }^{13} \mathrm{C} \mathrm{NMR}$ $\left(50.3 \mathrm{MHz}, \mathrm{CDCl}_{3}\right) \delta 20.3,33.1,45.7,122.3,130.2,130.9,136.3,139.6,158.2,204.2$. MS (EI) $\mathrm{m} / z$ (rel. intensity, \%): $164\left(\mathrm{M}^{+}, 70\right), 163\left(\mathrm{M}^{+}-\mathrm{H}, 100\right), 149\left(\mathrm{M}^{+}-\mathrm{CH}_{3}, 16\right), 145\left(\mathrm{M}^{+}-\mathrm{F}, 6\right)$; HRMS Cacld. for $\mathrm{C}_{10} \mathrm{H}_{9} \mathrm{FO}\left(\mathrm{M}^{+}\right)$164.0637; Found 164.0637.

\section{7-Chloro-3-methyl-1-indanone (5d)}<smiles>CC1CC(=O)c2c(Cl)cccc21</smiles>

Colorless oil, $\mathrm{R}_{f}=0.45$ [ethyl acetate: petroleum ether $\left(60-90{ }^{\circ} \mathrm{C}\right) 1: 15, \mathrm{v} / \mathrm{v}$, silica gel plate]; IR $(\mathrm{KBr}) v$ $\left(\mathrm{cm}^{-1}\right): 1720.0(\mathrm{C}=\mathrm{O}) ;{ }^{1} \mathrm{H}$ NMR $\left(200 \mathrm{MHz}, \mathrm{CDCl}_{3}\right) \delta 7.62-7.41(\mathrm{~m}, 3 \mathrm{H}), 3.60(\mathrm{ddq}, J=7.6,1.8,7.0 \mathrm{~Hz}$, $1 \mathrm{H}), 2.99(\mathrm{dd}, J=19.2,7.6 \mathrm{~Hz}, 1 \mathrm{H}), 2.40(\mathrm{dd}, J=19.2,1.8 \mathrm{~Hz}, 1 \mathrm{H}), 1.44(\mathrm{~d}, J=7.0 \mathrm{~Hz}, 3 \mathrm{H}) ;{ }^{13} \mathrm{C} \mathrm{NMR}$ $\left(50.3 \mathrm{MHz}, \mathrm{CDCl}_{3}\right) \delta 20.3,33.1,45.8,122.3,129.2,130.2,136.4,139.6,158.2,204.1$. MS (EI) $\mathrm{m} / \mathrm{z}$ (rel. intensity, \%): $180\left(\mathrm{M}^{+}, 20\right), 165\left(\mathrm{M}^{+}-\mathrm{CH}_{3}, 30\right), 145\left(\mathrm{M}^{+}-\mathrm{Cl}, 13\right), 115$ (100), 91 (7); HRMS Cacld. for $\mathrm{C}_{10} \mathrm{H}_{9} \mathrm{ClO}\left(\mathrm{M}^{+}\right)$180.0342; Found 180.0344 .

\section{7-Bromo-3-methyl-1-indanone (5e)}<smiles>CC1CC(=O)c2c(Br)cccc21</smiles>

Colorless oil, $\mathrm{R}_{f}=0.45$ [ethyl acetate: petroleum ether $\left(60-90{ }^{\circ} \mathrm{C}\right) 1: 15$, v/v, silica gel plate]; $\mathrm{IR}(\mathrm{KBr}) v$ $\left(\mathrm{cm}^{-1}\right): 1719.7(\mathrm{C}=\mathrm{O}){ }^{1} \mathrm{H}$ NMR $\left(200 \mathrm{MHz}, \mathrm{CDCl}_{3}\right) \delta$ 7.84-7.27 (m, 3H), 3.61 (ddq, $J=7.8,1.8,7.0 \mathrm{~Hz}$, $1 \mathrm{H}), 2.99(\mathrm{dd}, J=19.2,7.8 \mathrm{~Hz}, 1 \mathrm{H}), 2.40(\mathrm{dd}, J=19.2,1.8 \mathrm{~Hz}, 1 \mathrm{H}), 1.44(\mathrm{~d}, J=7.0 \mathrm{~Hz}, 3 \mathrm{H}) ;{ }^{13} \mathrm{C} \mathrm{NMR}$ $\left(50.3 \mathrm{MHz}, \mathrm{CDCl}_{3}\right) \delta 20.3,33.2,45.8,122.3,129.8,130.3,131.9,138.3,158.3,204.2$. MS (EI) $\mathrm{m} / z$ (rel. intensity, \%): $224\left(\mathrm{M}^{+}, 19\right), 209\left(\mathrm{M}^{+}-\mathrm{CH}_{3}, 33\right), 183\left(\mathrm{M}^{+}-\mathrm{CH}_{3} \mathrm{CHCH}_{2}, 100\right), 145\left(\mathrm{M}^{+}-\mathrm{Br}, 13\right)$; HRMS Cacld. for $\mathrm{C}_{10} \mathrm{H}_{9} \mathrm{BrO}\left(\mathrm{M}^{+}\right)$223.9837; Found 223.9835 .

\section{REFERENCES}

1. Gagnier, S. V.; Larock, R. C. J. Am. Chem. Soc. 2003, 125, 4804-4807.

2. Kemp, W.; Spanswick, J. J. Chem. Soc. Perkin Trans. 1. 1972, 1, 151-155.

3. Allen, J. M.; Johnston, K. M.; Jones, J. F.; Shotter, R. G. Tetrahedron. 1977, 33, 2083-2087.

4. Pines, S. H.; Douglas, A. W. J. Am. Chem. Soc.1976, 98, 8119-8125.

5. Kerr, C. A.; Rae, I. D. Aust. J. Chem. 1978, 31, 341-346. 
6. Sircar, I.; Duell, B. L.; Cain, M. H.; Burke, S. E.; Bristol, J. A. J. Med. Chem. 1986, 29, 2142-2148.

7. Smith, C. E.; Williamson, W. R. N.; Cashin, C. H.; Kitchen, E. A. J. Med. Chem. 1979, 22, 1464-1469.

8. Boykin, D. W.; Hertzler, R. L.; Delphon, J. K.; Eisenbraun, E. J. J. Org. Chem. 1989, 54, 1418-23.

9. Boegesoe, K. P.; Arnt, J.; Hyttel, J.; Pedersen, H. J. Med. Chem. 1993, 36, 2761-2770. 


\section{5-Isopropyl-3-methyl-1-indanone (4g)}<smiles>CC(C)c1ccc2c(c1)C(C)CC2=O</smiles>

containing a little $\mathbf{4 a}$
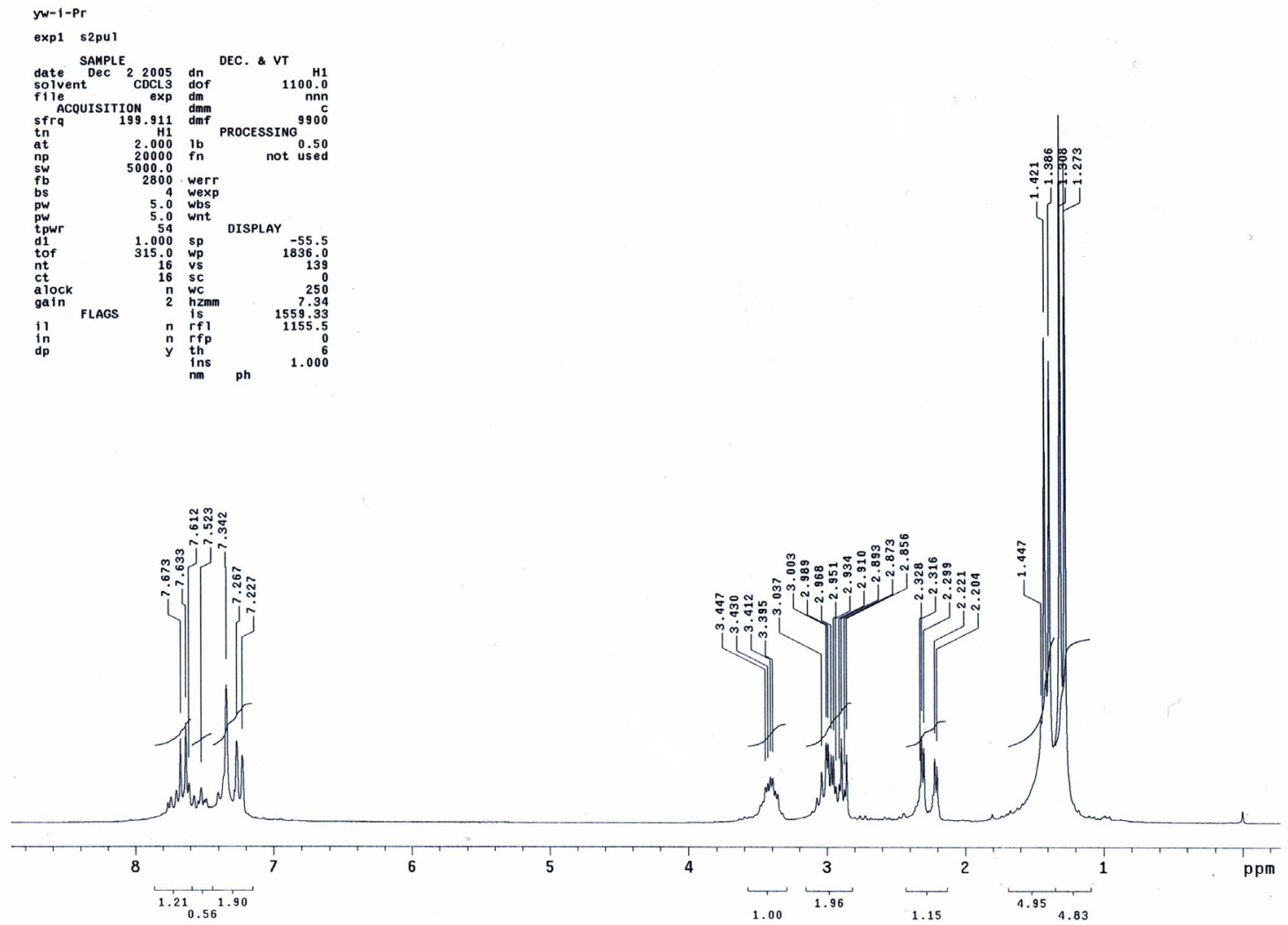

$4 g$ containing a little $4 a$

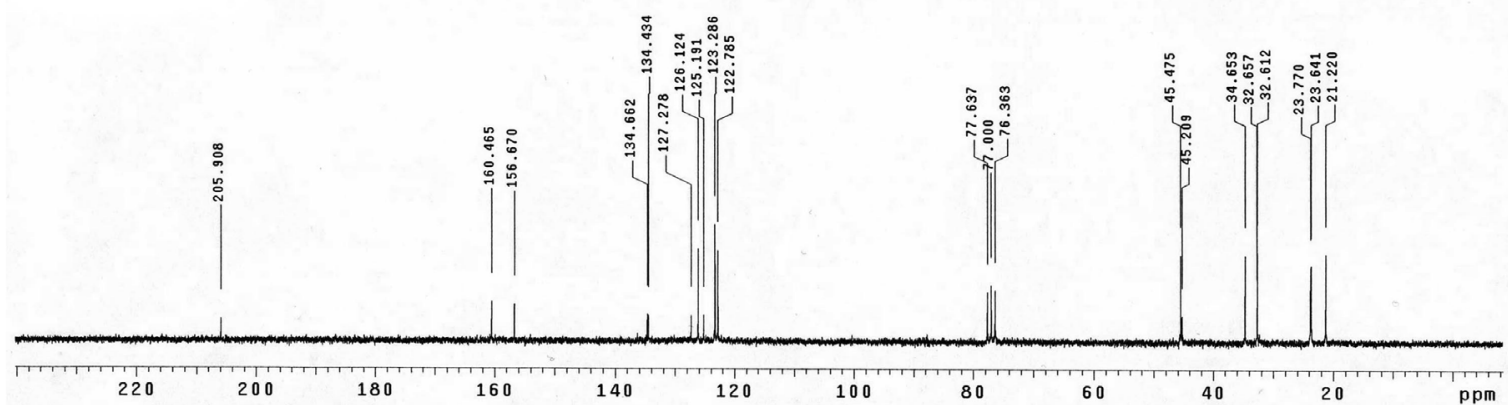

${ }^{13} \mathrm{C}$ NMR spectrum of $\mathbf{4 a}$

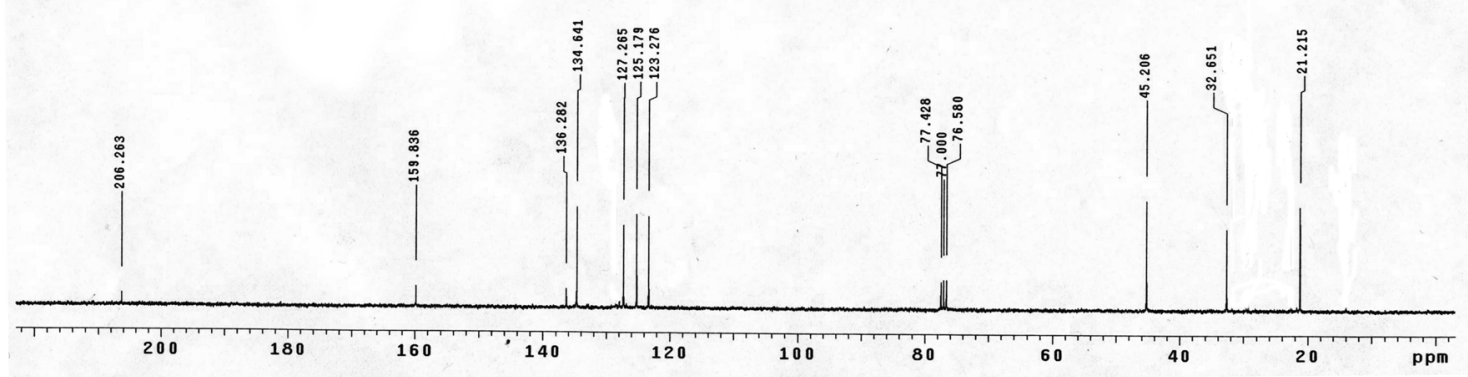




\section{5,6-Dichloro-3-methyl-1-indanone (4k)}
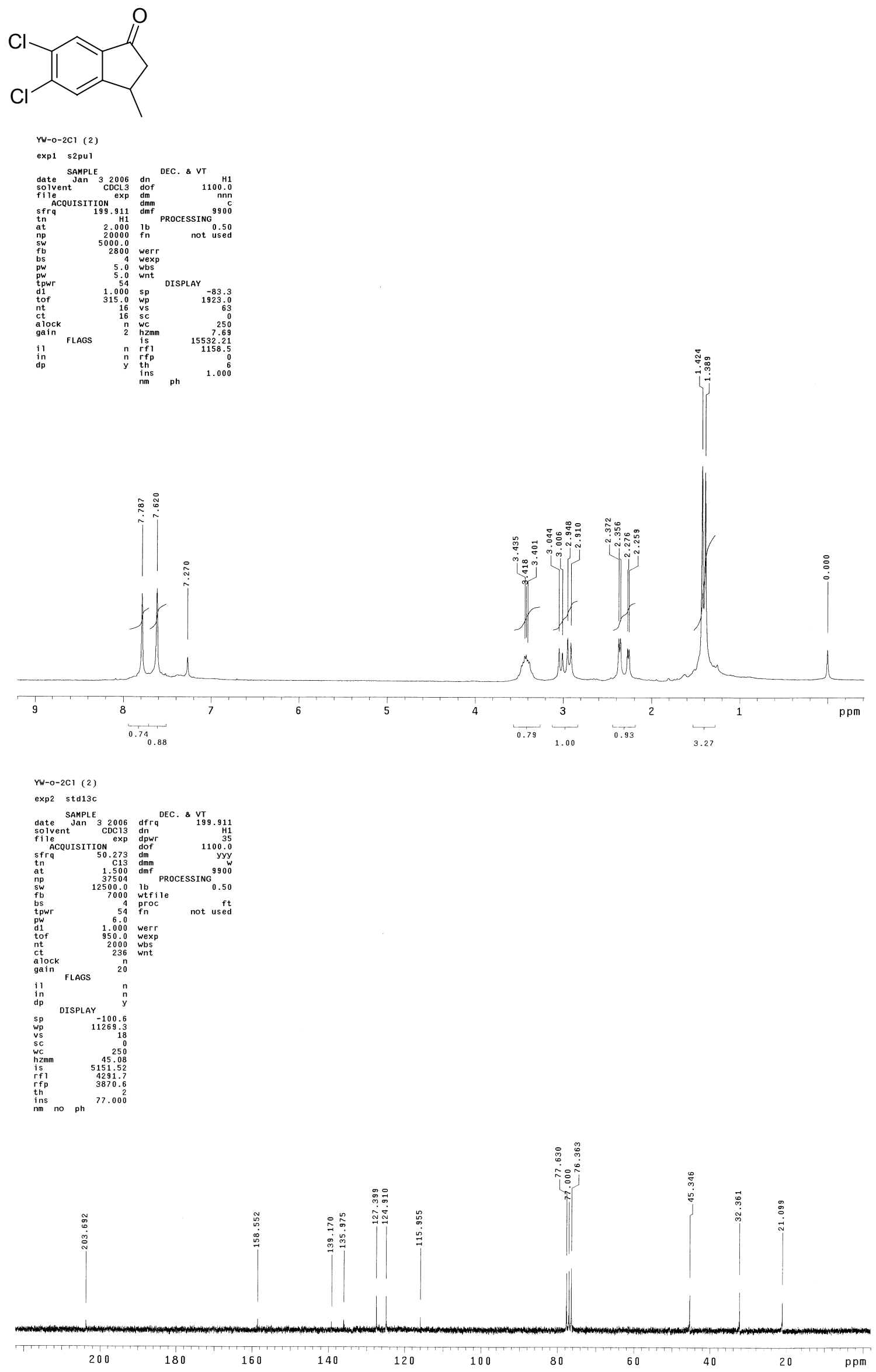


\section{4,5-Dichloro-3-methyl-1-indanone (4k')}<smiles>CC1CC(=O)c2ccc(Cl)c(Cl)c21</smiles>

YW-o2C1 (1)
exp1 s2pu
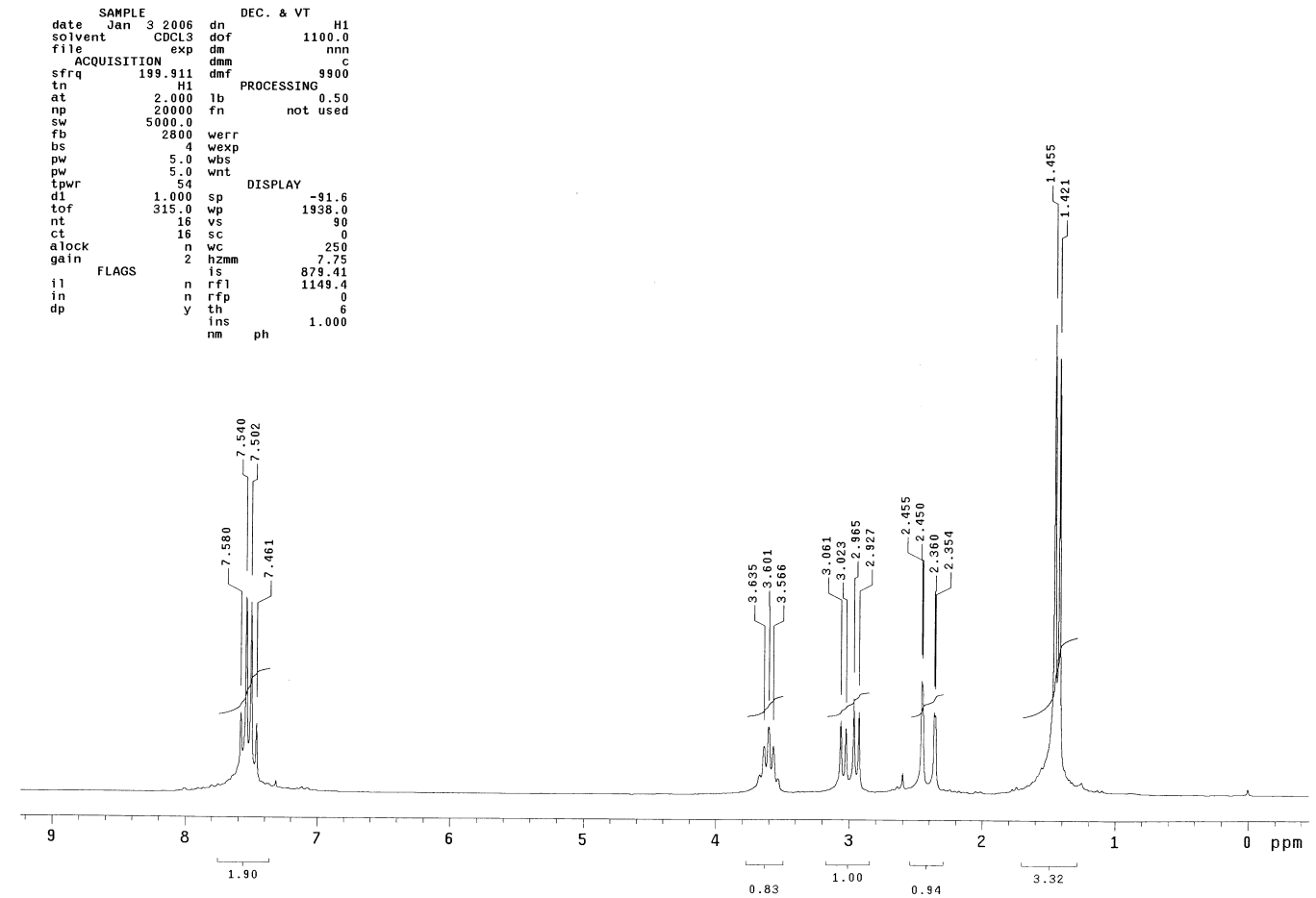

YW-O2C1 (1)

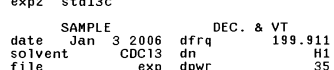

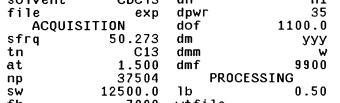

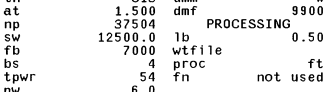

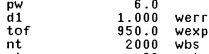

$\begin{array}{lr}c t & 36 \\ \text { alock } & n \\ \text { gain } & n \\ & 20\end{array}$

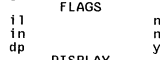

$\begin{array}{rr}\text { sp } & \text { DISPLAY } \\ \text { wp } & -136.1 \\ \text { vs } & 11372.7 \\ \text { we } & \end{array}$

$\begin{array}{lr}\text { sc } & 250 \\ \text { wC } & 250 \\ \text { hrmm } & 45.49 \\ \text { is } & 5151.52 \\ \text { rf } & 4295.9 \\ \text { rfp } & 3870.6 \\ \text { th } & \text { th }\end{array}$

ns 77.000

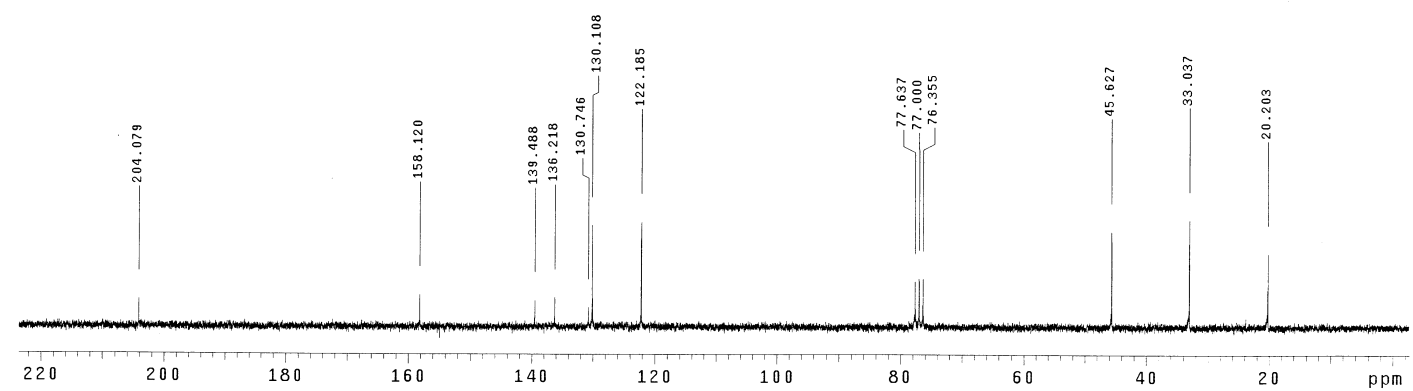


5-Fluoro-3-phenyl-1-indanone ( $4 \mathrm{~m})$<smiles>O=C1CC(c2ccccc2)c2cc(F)ccc21</smiles>

Yw-CIn-F 22-24

exp1 52pul

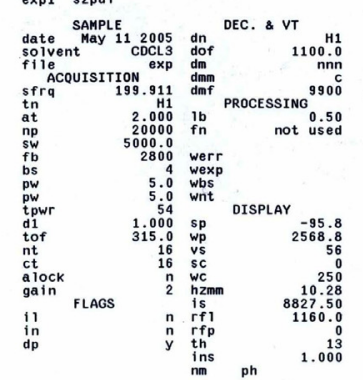

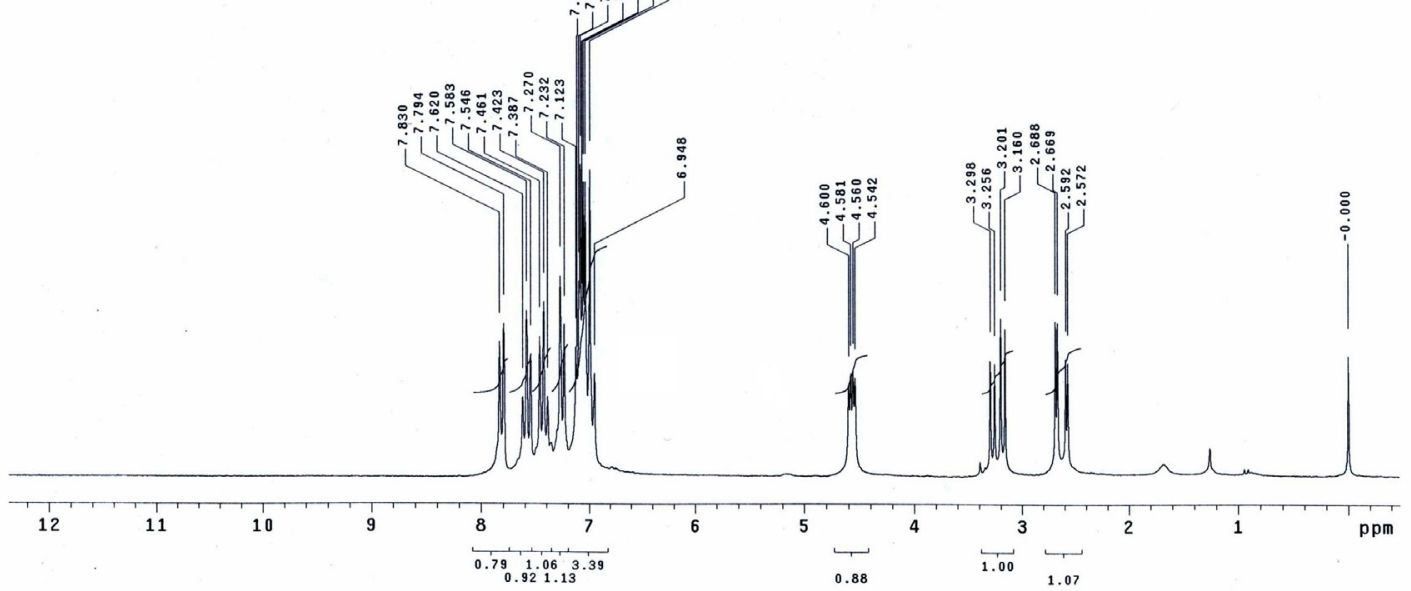

YW-cin-F 22-24

exp2 std13c

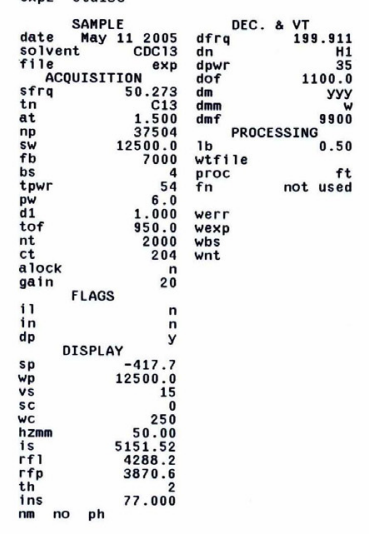

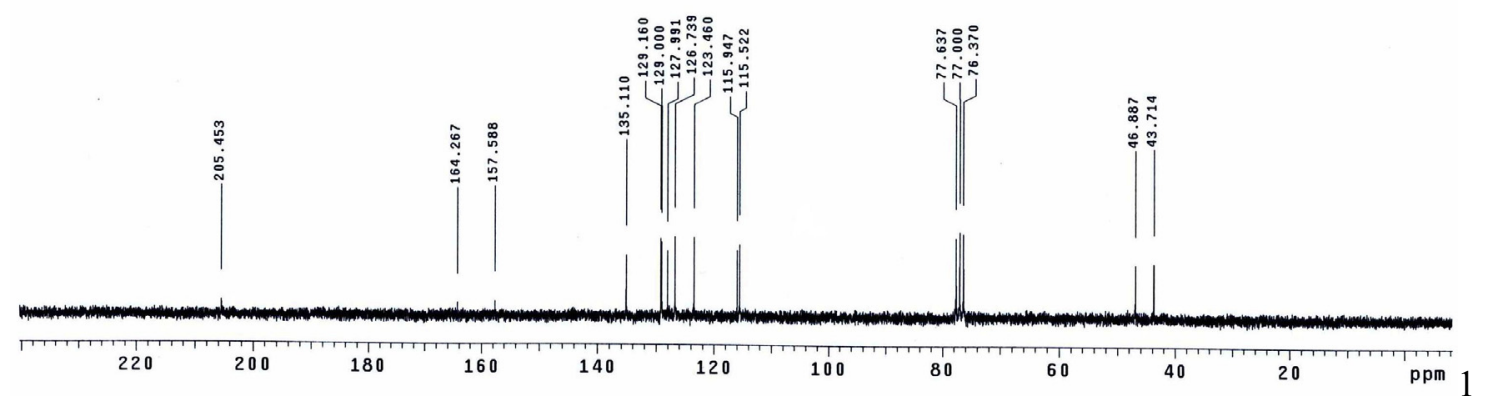


5-Bromo-3-phenyl-1-indanone (4o)<smiles>O=C1CC(c2ccccc2)c2cc(Br)ccc21</smiles>

YW-Cin-Br 25-27

exp1 s2pul

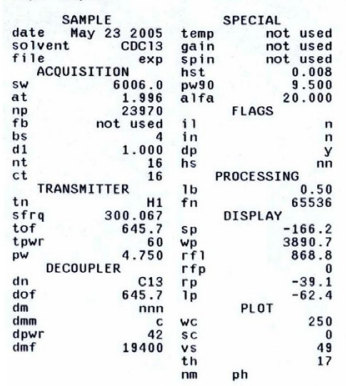

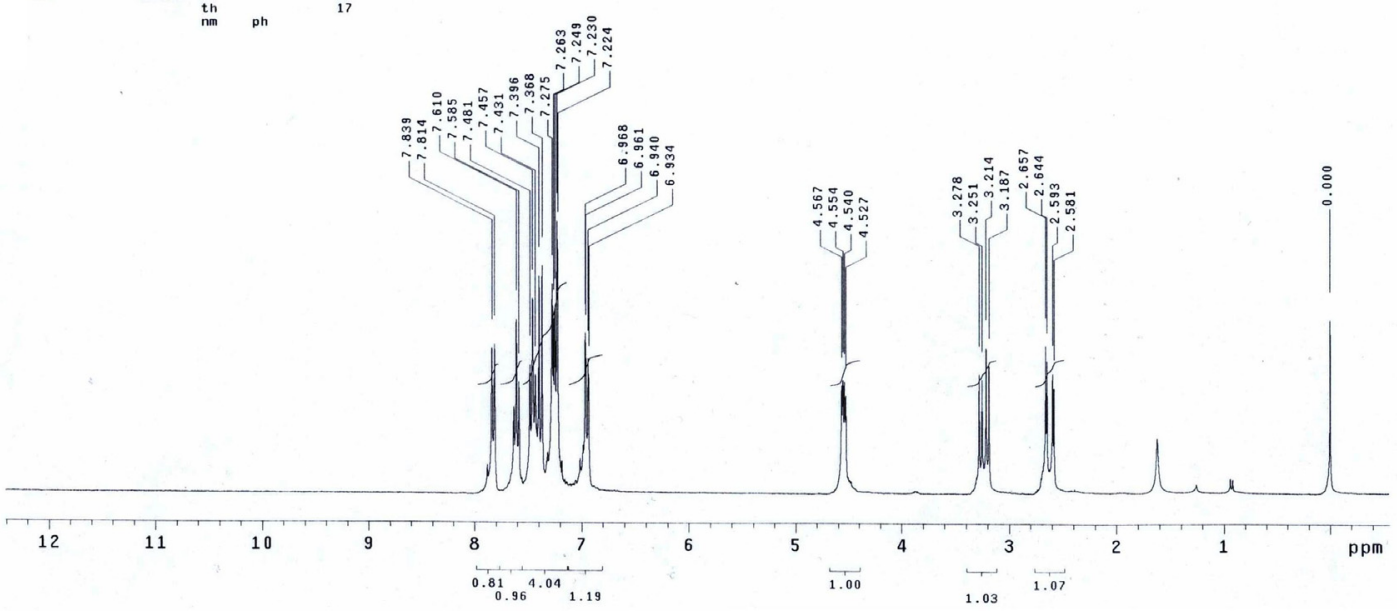

Ww-Cin-Br 25-27

exp2 std13c
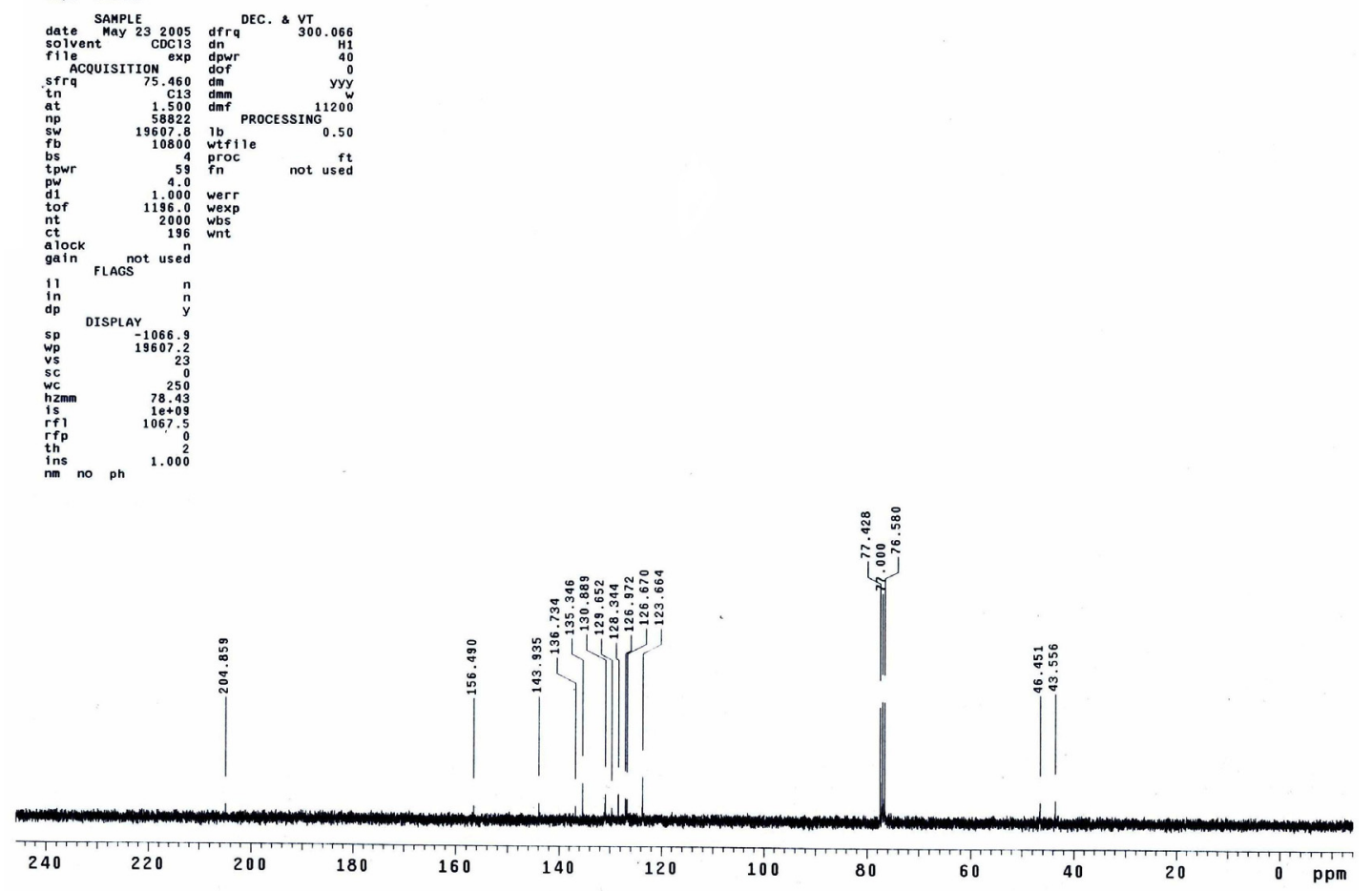
7-Fluoro-3-methyl-1-indanone (5c)<smiles>CC1CC(=O)c2c(F)cccc21</smiles>

$$
\text { yw-o-F }
$$$$
\text { exp1 s2pul }
$$$$
\begin{aligned}
& \text { Sate } \\
& \text { Solvent } \\
& \text { DeC } 22005 \text { an DEC. \& V V }
\end{aligned}
$$

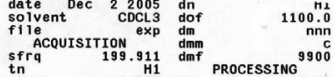

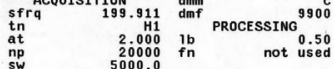

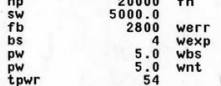

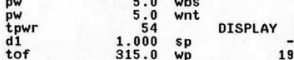

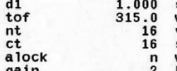$$
\text { gain FLAGS }
$$$$
\operatorname{lin}_{\substack{n \\ \text { di }}}
$$

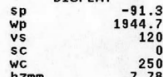

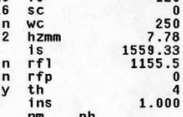

nm ph

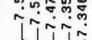
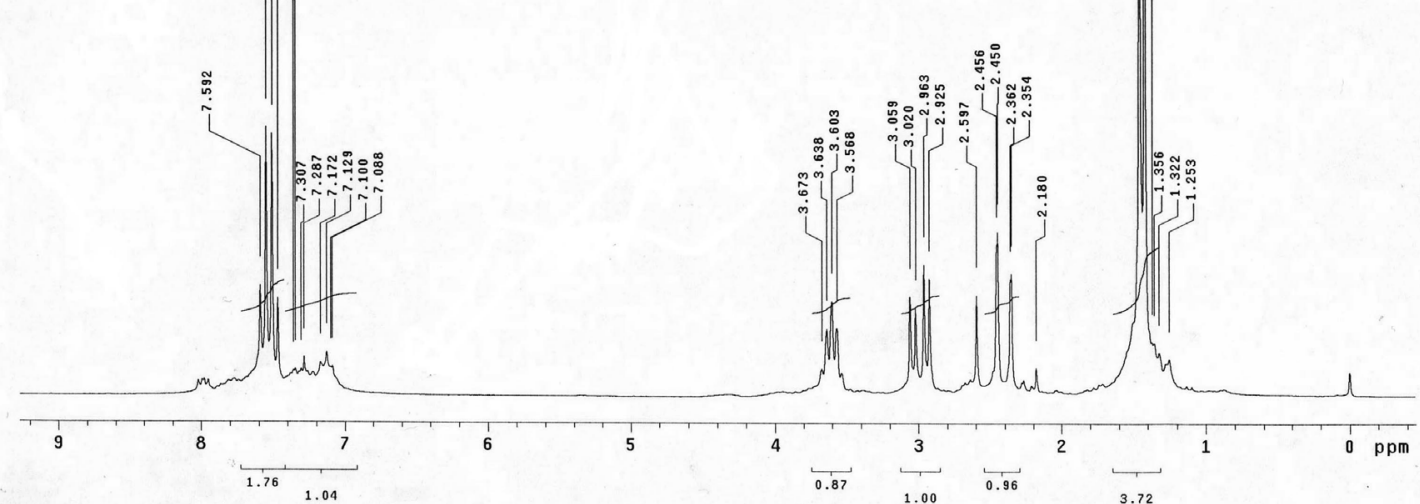

yw-o-f

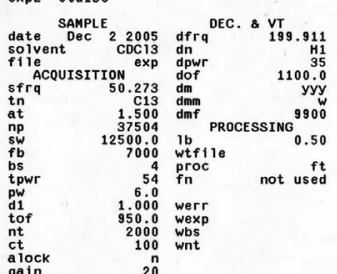

20

$\begin{array}{lll}\text { in } & \text { FLAGS } & n \\ \text { dip } & & n \\ & \text { DISPLAY } & y \\ & & \end{array}$

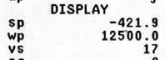

$\begin{array}{lr}\text { sc } & 50 \\ \text { wc } & 250 \\ \text { hrmm } & 55.00 \\ \text { is } & 515.52 \\ \text { rf } & 52.52 \\ \text { rfp } & 4292.4 \\ \text { th } & 3870.6 \\ \text { th } & 27\end{array}$

the
thp
ins

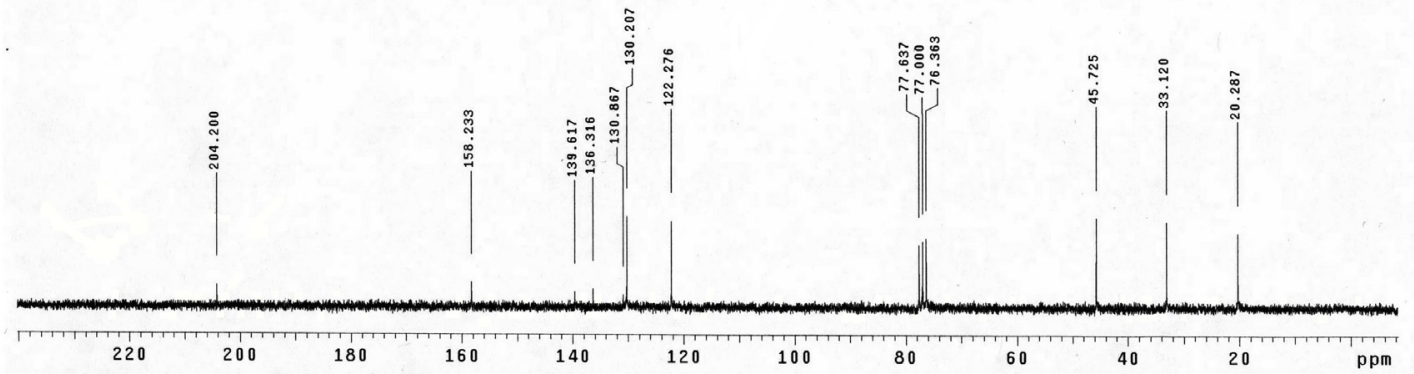


7-Chloro-3-methyl-1-indanone (5d)<smiles>CC1CC(=O)c2c(Cl)cccc21</smiles>

exp1 s2

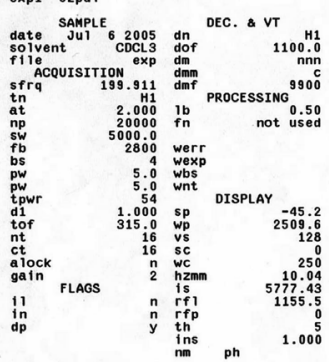

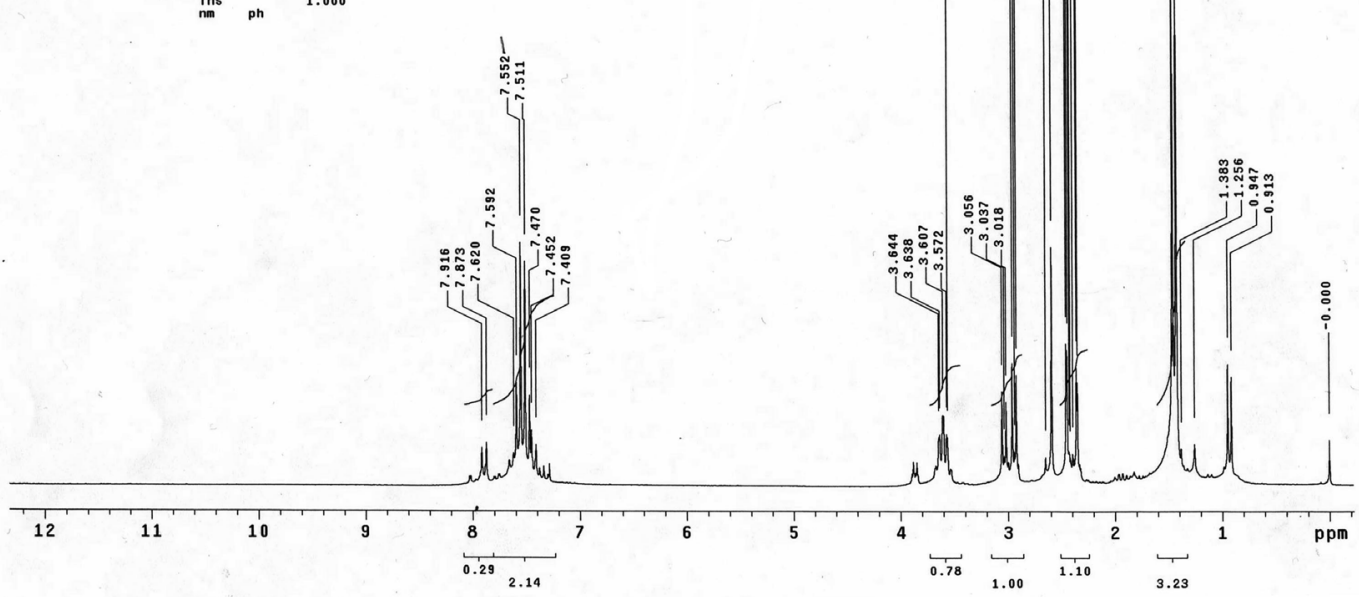

YW-C1-1

exp2 std13c

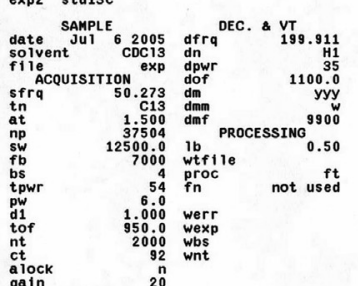

$\prod_{\substack{11 \\ \text { in }}}$

dp DISPLAY

$\begin{array}{lr}\text { sp } & \text { OSPLAY } \\ \text { wp } \\ \text { vs } & 11341.8 \\ \text { sc } & 17 \\ \text { we } & \text { if }\end{array}$

$\begin{array}{lr}\text { wc } & 250 \\ \text { hzmm } & 45.37 \\ \text { is } & 5151.52 \\ \text { rf } & 421.5 \\ \text { rfp } & 3870.3 \\ \text { th } & 38 \\ & 72.000\end{array}$

th
ins
nm no ph 72.00

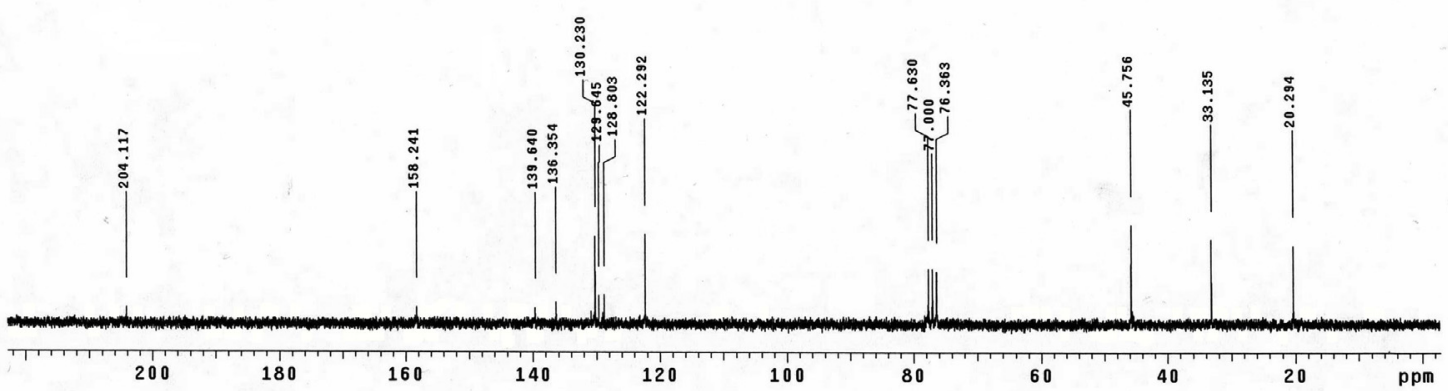


7-Bromo-3-methyl-1-indanone (5e)
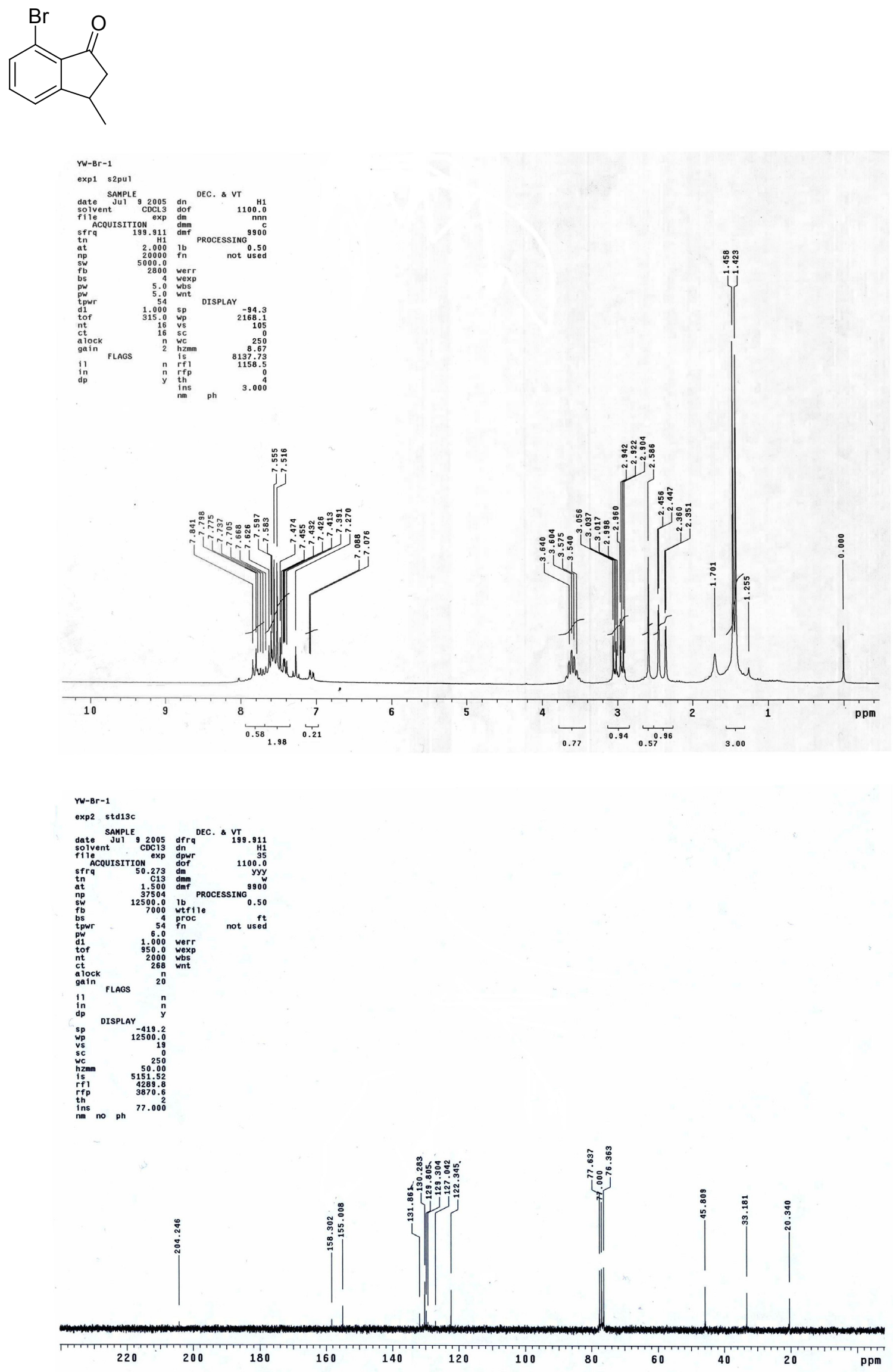
7-Bromo-3-phenyl-1-indanone (5o)<smiles>O=C1CC(c2ccccc2)c2cccc(Br)c21</smiles>

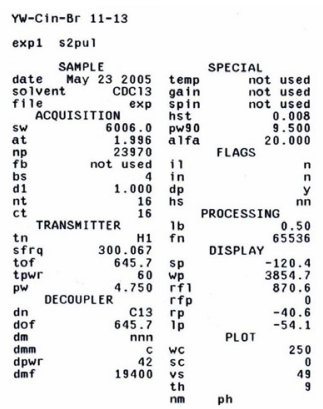

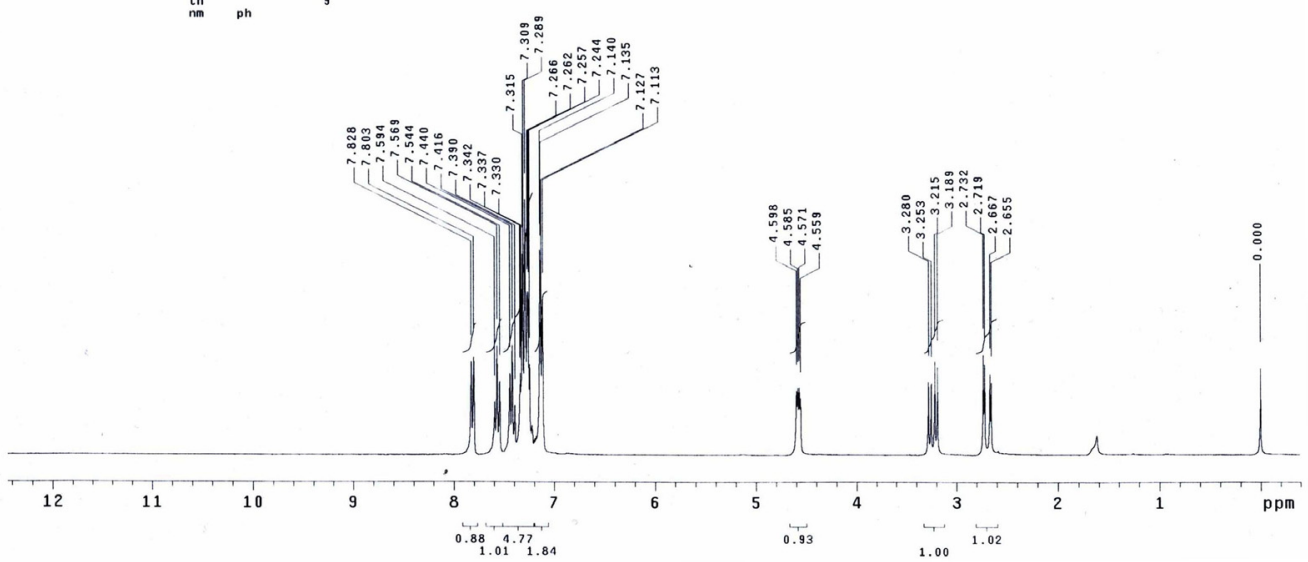

YW-Cin-Br 11-13

exp2 std13c
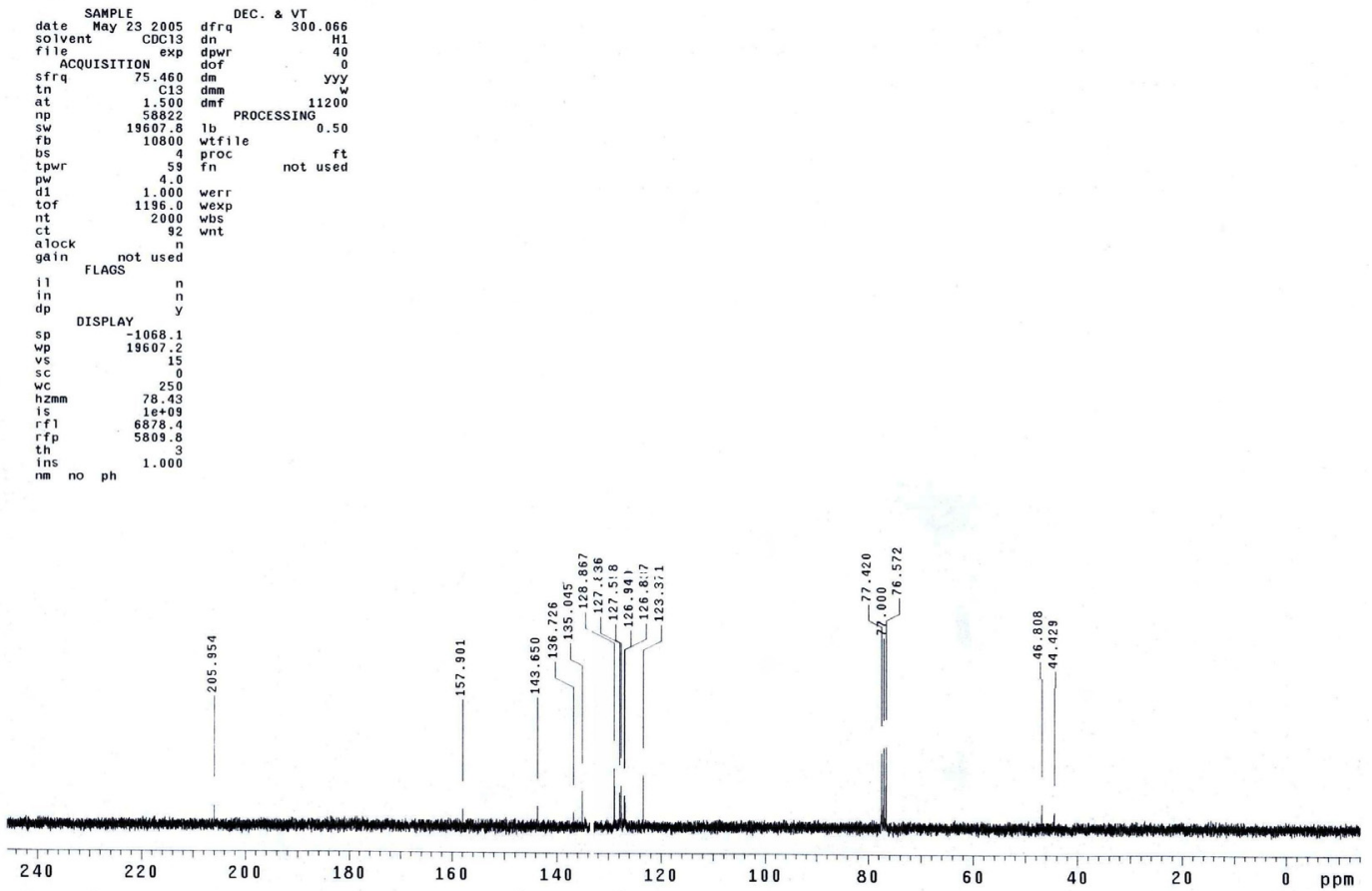
NOE spectrum of 4,5-Dichloro-3-methyl-1-indanone (4k')<smiles>CC1CC(=O)c2ccc(Cl)c(Cl)c21</smiles>

No obvious NOE was observed.

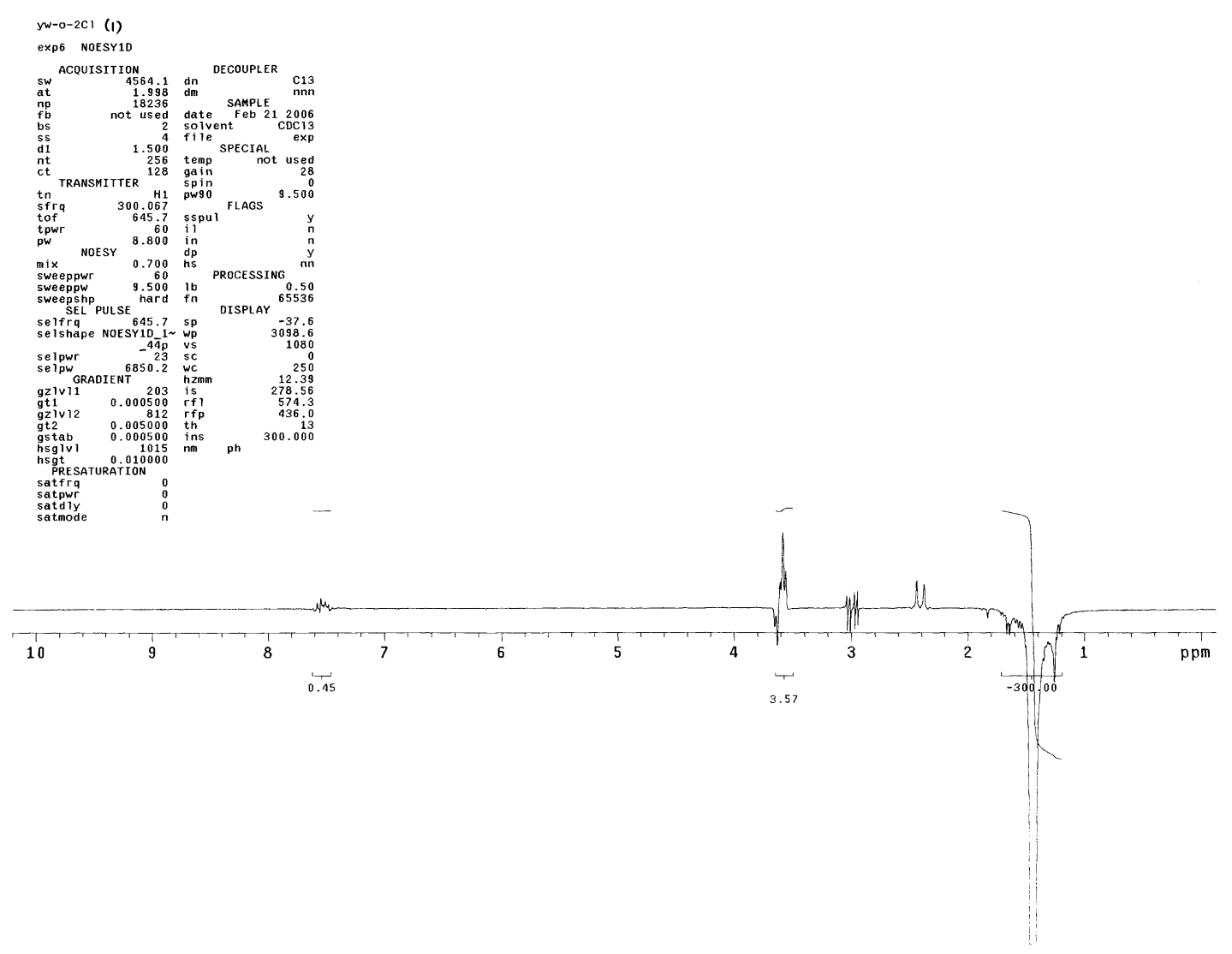

